\title{
The beam breakup instability in quadrupole and solenoidal electron-beam transport systems
}

\author{
R. A. Bosch, a) P. R. Menge, and R. M. Gilgenbach \\ Intense Energy Beam Interaction Laboratory, Nuclear Engineering Department, \\ The University of Michigan, Ann Arbor, Michigan 48109-2104
}

(Received 27 August 1991; accepted for publication 19 December 1991)

\begin{abstract}
Dispersion relations are derived to determine the growth rate, dominant wavelength, and group velocity of disturbances caused by the beam breakup instability. Considerations include weak and strong focusing, $x-y$ coupling in solenoidal transport, the spacing of accelerator cavities, and periodically pulsed beams. Beam breakup growth is minimum when the cavity spacing equals an integral number of half-betatron wavelengths for quadrupole focusing, and an integral number of betatron wavelengths for solenoidal focusing. Minimum growth is also found for periodic pulses separated by an integral number of half-periods of the $\mathrm{TM}_{110}$ cavity mode. Expressions for beam breakup growth at the minima are obtained.
\end{abstract}

\section{INTRODUCTION}

The acceleration and transport of high-brightness electron and ion beams are subject to disruption by the beam breakup (BBU) instability. ${ }^{1-7}$ This instability results from coupling of transverse beam oscillations and a nonaxially symmetric mode of the accelerating structure. With cylindrical pillbox accelerating cavities, the $\mathrm{TM}_{110}$ mode produces the maximum instability growth.

In the regenerative BBU instability, upstream propagation of the $\mathrm{TM}_{110}$ mode provides feedback for amplification within a single accelerator section. ${ }^{2}$ In the cumulative, or multisection, BBU instability, the $\mathrm{TM}_{110}$ modes of different accelerator sections are coupled only by the passage of the electron (or ion) beam. In this article, we study the cumulative BBU instability in linear focusing systems. In quadrupole focusing systems or systems containing certain nonaxisymmetric optical elements (e.g., magnetic sector field and edge focusing), the $x$ and $y$ directions of motion are not coupled. ${ }^{8}$ With solenoidal transport, the BBU instability is affected by the coupling of the $x$ and $y$ directions of transverse motion. ${ }^{9}$

Our approach is to examine the BBU dispersion relations. This is a practical approach motivated by ongoing experiments: ${ }^{10,11}$ the wavelength, $e$ folding length, and group velocity of a BBU disturbance are directly obtainable from the dispersion relation. The dispersion relation may also be used in a study of the response to initial conditions, and calculation of beam offset versus time at a fixed position.?

We first consider continuum models of quadrupole and solenoidal transport, applicable when the accelerating cavity spacing is small compared to beam breakup scale lengths and the beam current is constant. Our results agree with several prior calculations.

We then consider the case of finite accelerator cavity spacing, treating the cavity forces as periodic impulses. For cavity spacing that is an integral number of half-betatron

\footnotetext{
'Present address: Synchrotron Radiation Research Center, Hsinchu Science-based Industrial Park, Hsinchu, Taiwan, Republic of China.
}

wavelengths with quadrupole transport, or an integral number of betatron wavelengths with solenoidal transport, BBU growth vanishes. When the finite cavity length is considered, BBU growth for these cavity spacings is calculated and found to be finite.

Finally, we consider the case of a pulsed beam. For pulses of vanishing pulse length separated by an integral multiple of one-half the $\mathrm{TM}_{110}$ period of the accelerating cavities, BBU growth vanishes. When a finite pulse length is considered, BBU growth no longer vanishes completely at these pulse separations. An expression for BBU growth with finite pulse length is presented.

\section{THE ONE-DIMENSIONAL CONTINUUM MODEL FOR QUADRUPOLE TRANSPORT}

We consider a beam transport system with periodically spaced cylindrical pillbox accelerating cavities. Transverse motions in the $x$ and $y$ directions are not coupled with quadrupole transport. Beam displacements in the $x$ direction excite one polarization of the $\mathrm{TM}_{110}$ wavemode in the accelerator cavities, producing a magnetic field in the $y$ direction on axis. The resultant $\mathbf{v} \times \mathbf{B}$ force is in the $x$ direction. Consequently, the $x$ and $y$ directions of motion are not coupled in the BBU instability.

For quadrupole transport, the BBU instability has been studied previously using a one (transverse) -dimensional coupled-mode description. ${ }^{3,7}$ The one-dimensional coupled-mode equations are

$$
\begin{aligned}
& \frac{d}{d t} \gamma \frac{d x}{d t}+\gamma \omega_{c}^{2} x=a, \\
& \left(\frac{\partial^{2}}{\partial t^{2}}+\frac{\omega_{0}}{Q} \frac{\partial}{\partial t}+\omega_{0}^{2}\right) a=2 \gamma \omega_{0}^{4} \in x,
\end{aligned}
$$

where $\gamma=\left(1-\beta^{2}\right)^{-1 / 2}$ is the relativistic mass factor, $\omega_{c}$ is the betatron angular frequency, and $d / d t=\partial / \partial t+v \partial /$ $\partial z$ is the convective derivative. Equation (1a) describes the acceleration of the beam by the focusing field and the magnetic field $B_{y}$ of the $\mathrm{TM}_{110}$ mode. Equation (1b) describes excitation of the $\mathrm{TM}_{110}$ mode with angular frequency $\omega_{0}$ 
$\left(\omega_{0}>0\right)$ and quality factor $Q$ by the transverse beam disturbance. The quantity $\epsilon$ is the dimensionless coupling constant of the $e$ beam to the $\mathbf{T M}_{110}$ mode, given by ${ }^{12}$

$$
\epsilon=0.422 \frac{l}{L} \frac{I}{17 k A} \frac{\beta}{\gamma},
$$

where $l$ is the length of the microwave cavities, $L$ is their spacing, and $I$ is the beam current magnitude $(I>0)$. The coupling constant is related to the transverse impedance of Ref. 4 by

$$
\epsilon=\frac{c}{60 \omega_{0} L} \frac{Z_{\perp} \text { (ohms) }}{Q} \frac{I}{17 k A} \frac{\beta}{\gamma} .
$$

For a coasting beam, $v, \gamma$, and $\epsilon$ are constant. Assuming a disturbance of the form $e^{i \omega t-i k z}$, Eqs. (1) and (2) yield the dispersion relation

$$
\Omega^{2}-\omega_{c}^{2}+\Gamma=0,
$$

where

$$
\Omega=\omega-v k \quad \text { and } \Gamma=\frac{2 \omega_{0}^{4} \epsilon}{-\omega^{2}+\omega_{0}^{2}+i \omega \omega_{0} / Q} .
$$

For real, positive values of $\omega$, the magnitude of $\Gamma$ is maximum for $\omega \cong \omega_{0}$, where $\Gamma\left(\omega_{0}\right)=-2 i \omega_{0}^{2} \epsilon Q$.

Scaling laws can be determined from the dispersion relation by considering the case where $\omega$ is real and positive. The $e$ folding length of the instability is given by

$$
l_{e}=\left[\left.\operatorname{Im}(k)\right|_{\omega_{\max }}\right]^{-1},
$$

where $\omega_{\max }$ is the frequency giving the largest value of $\operatorname{Im}(k)$. In this model of the BBU instability, $\omega_{\max } \cong \omega_{0}$, with $\omega_{0}(1-1 / 2 \sqrt{3} Q) \leqslant \omega_{\max } \leqslant \omega_{0}$. The wavelength of maximum growth is

$$
\lambda=\frac{2 \pi}{\left.\operatorname{Re}(k)\right|_{\omega_{\max }}} .
$$

The group velocity of a BBU disturbance dominated by the frequency $\omega_{\max }$ is given by

$$
v_{g}=\left[\left.\operatorname{Re}\left(\frac{\partial k}{\partial \omega}\right)\right|_{\omega_{\max }}\right]^{-1}=\left(\left.\frac{\partial k}{\partial \omega}\right|_{\omega_{\max }}\right)^{-1} .
$$

Consider a disturbance excited at $(z, t)=(0,0)$ and dominated by frequency $\omega_{\max }$. The time $t_{\max }$, at which the disturbance peaks at fixed $z$, is related to $z$ by $t_{\max }=z / v_{g}$, while the disturbance amplitude grows as $e^{z / l_{e}}=e^{\operatorname{Im}(k) z}$.

For sufficiently weak focusing that $\left|\Gamma\left(\omega_{0}\right)\right|$ $=2 \omega_{0}^{2} \epsilon Q>\omega_{c}^{2}$, the dispersion relation reduces to

$$
\Omega^{2}+\Gamma=0 \text {. }
$$

For $Q>1$, maximum growth occurs for $\omega_{\max }=\omega_{0}$ $[1-(1 / 2 \sqrt{3} Q)]$, for which

$$
\begin{aligned}
\left.k\right|_{\omega_{\max }}= & \frac{1}{v}\left[\omega_{0}\left(1-\frac{1}{2 \sqrt{3} Q}\right)\right. \\
& \left. \pm \omega_{0} \epsilon^{1 / 2} Q^{1 / 2} \frac{3^{3 / 4}}{2}\left(i+3^{-1 / 2}\right)\right],
\end{aligned}
$$

$$
\left.\frac{\partial k}{\partial \omega}\right|_{\omega_{\max }}=\frac{1}{v}\left(1 \pm \epsilon^{1 / 2} Q^{3 / 2} \frac{3^{3 / 4}}{2}\right) .
$$

The mode with $\operatorname{Im}(k)>0$ propagates downstream as it grows.

For sufficiently strong focusing that $\left|\Gamma\left(\omega_{0}\right)\right|<\omega_{c}^{2}$, the $e$ folding length given by Eq. (8) exceeds the betatron wavelength. In this strong focusing regime, the dispersion relation reduces to

$$
\Omega= \pm\left(\omega_{c}^{2}-\Gamma\right)^{1 / 2} \cong \pm\left[\omega_{c}-\left(\Gamma / 2 \omega_{c}\right)\right] .
$$

Maximum instability growth is obtained at $\omega_{\max }=\omega_{0}$, for which

$$
\begin{aligned}
& \left.k\right|_{\omega_{\max }}=\frac{1}{v}\left[\omega_{0} \pm\left(\omega_{c}+\frac{i \omega_{0}^{2} \epsilon Q}{\omega_{c}}\right)\right], \\
& \left.\frac{\partial k}{\partial \omega}\right|_{\omega_{\max }}=\frac{1}{v}\left(1 \pm 2 \epsilon Q^{2} \frac{\omega_{0}}{\omega_{c}}\right) .
\end{aligned}
$$

Because $\operatorname{Im}(k) \propto \epsilon \propto 1 / L$, the growth per cavity in the strong focusing regime is independent of the cavity spacing. For weak and strong focusing, the $e$ folding lengths and group velocities obtained from Eqs. (8), (9), (11), and (12) agree with asymptotic calculations of the response to an impulse ${ }^{7}$ [see Eq. (12a) for weak focusing and Eq. (12b) for strong focusing in Ref. 7]. In the strong focusing regime, these results are also in agreement with the approach of Ref. 4, if a transfer matrix for quadrupole transport is employed.

\section{A TWO-DIMENSIONAL CONTINUUM MODEL FOR SOLENOIDAL TRANSPORT}

In solenoidal transport systems, transverse motions in the $x$ and $y$ directions are coupled by the $\mathbf{v} \times \mathbf{B}$ force. To model this case, we consider a two (transverse) -dimensional continuum description of the beam motion, ${ }^{9}$

$$
\begin{aligned}
& \frac{d}{d t} \gamma \frac{d x}{d t}+\gamma \omega_{c} \frac{d y}{d t}=a_{x}, \\
& \frac{d}{d t} \gamma \frac{d y}{d t}-\gamma \omega_{c} \frac{d x}{d t}=a_{y},
\end{aligned}
$$

where $\omega_{c}=e B / m \gamma$ is the relativistic cyclotron frequency. The $\mathrm{TM}_{110}$ modes of cylindrical pillbox accelerating cavities are excited by $e$-beam displacements according to

$$
\begin{aligned}
& \left(\frac{\partial^{2}}{\partial t^{2}}+\frac{\omega_{0}}{Q} \frac{\partial}{\partial t}+\omega_{0}^{2}\right) a_{x}=2 \gamma \omega_{0}^{4} \epsilon x, \\
& \left(\frac{\partial^{2}}{\partial t^{2}}+\frac{\omega_{0}}{Q} \frac{\partial}{\partial t}+\omega_{0}^{2}\right) a_{y}=2 \gamma \omega_{0}^{4} \epsilon y .
\end{aligned}
$$

Equations (13) and (14) yield the dispersion relation

$$
\left(\Omega^{2}-\omega_{c} \Omega+\Gamma\right)\left(\Omega^{2}+\omega_{c} \Omega+\Gamma\right)=0 .
$$

While Eq. (15) is easily obtained directly from Eqs. (13) and (14), an alternative approach is to consider the helical Larmor frame: $x^{\prime}=x \cos \theta+y \sin \theta, y^{\prime}=y \cos \theta$ $-x \sin \theta$, where $\theta=\omega_{c} z / 2 v$. This frame twists at one- 


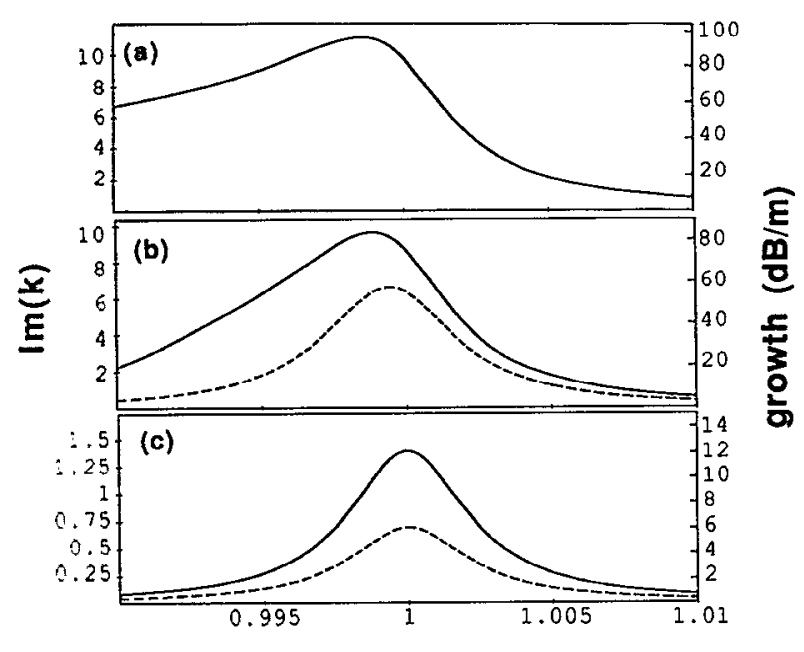

$\omega / \omega_{\circ}$

FIG. 1. The spatial growth rate $\operatorname{Im}(k)$ is graphed as a function of normalized angular frequency $\omega / \omega_{0}$, of the beam breakup wave. Curves are shown for the one-dimensional continuum model (dashed line) and the two-dimensional continuum model (solid line). The parameters used are kinetic energy $=700 \mathrm{keV}$, current $=100 \mathrm{~A}, \mathrm{TM}_{110}$ mode frequency $=2.5$ $\mathrm{GHz}, Q=200$, and $l / L=0.15$. (a) Growth rates in the weak focusing regime, with a betatron wavelength of $5 \mathrm{~m}$, and $\left|\Gamma\left(\omega_{0}\right)\right| / \omega_{c}^{2}=100$. In the weak focusing regime both models coincide, and the two curves lie atop each other. Note that the growth rate peaks at $\omega=\omega_{\max }$ $=\omega_{0}[1-(1 / 2 \sqrt{3} Q)]$. (b) Growth rates for a case intermediate to the weak and strong focusing regimes, with a betatron wavelength of $0.5 \mathrm{~m}$, and $\left|\Gamma\left(\omega_{0}\right)\right| / \omega_{c}^{2}=1$. (c) Growth rates in the strong focusing regime, with a betatron wavelength of $5 \mathrm{~cm}$, and $\left|\Gamma\left(\omega_{0}\right)\right| / \omega_{c}^{2}=0.01$. Note that the growth rate peaks at $\omega=\omega_{\max }=\omega_{0}$.

half the rate of electron trajectories. In this frame, Eqs. (13) separate into two equations identical to Eq. (1a) with $\omega_{c} \rightarrow \omega_{c} / 2$. Because the coordinate transformation is not time dependent, Eqs. (14) also hold in the helical frame. Consequently, the quadrupole focusing dispersion relation with $\omega_{c} \rightarrow \omega_{c} / 2$ describes solenoidal focusing in the helical frame. Letting $\Omega \rightarrow \Omega-\omega_{c} / 2$ or $\Omega \rightarrow \Omega+\omega_{c} / 2$ (i.e., $k \rightarrow k \pm \omega_{c} / 2 v$ ) gives the corresponding laboratory frame dispersion relation.

For weak focusing with $\left|\Gamma\left(\omega_{0}\right)\right|>\omega_{c}^{2}$, Eq. (15) reduces to the one-dimensional results of Eq. (7). For strong focusing with $\left|\Gamma\left(\omega_{0}\right)\right|<\omega_{c}^{2}$, Eq. (15) reduces to

$$
\begin{aligned}
& \Omega= \pm\left[\omega_{c}-\left(\Gamma / \omega_{c}\right)\right], \\
& \Omega= \pm\left(\Gamma / \omega_{c}\right) .
\end{aligned}
$$

Comparing with Eq. (11), we find that a strong solenoidal focusing system with cylindrical pillbox cavities has twice the BBU growth of a quadrupole focusing system with the same betatron wavelength.

The $e$ folding length and group velocity in the strong focusing regime are in agreement with Ref. 4 . In a previous article, ${ }^{9}$ we incorrectly stated that our results disagreed with Ref. 4 by a factor of 2 , as a result of incorrectly relating the coupling constant $\epsilon$ to the transverse impedance. The correct relation is given by $\mathrm{Eq} .(2 \mathrm{~b})$.

In Fig. 1, we plot the growth rate versus $\omega$ for the oneand two-dimensional models [Eqs. (3) and (15)]. The growth rate is plotted in units of $d B / m$, where growth $(\mathrm{dB} / \mathrm{m})=\left(20 \log _{10} e\right) \operatorname{Im}(k)\left(\mathrm{m}^{-1}\right)$. Figure $1(\mathrm{a})$ describes the weak focusing regime with $\left|\Gamma\left(\omega_{0}\right)\right| / \omega_{c}^{2}$ $=100$. The two models give nearly identical results, and the peak growth occurs at $\omega_{\max }=\omega_{0}[1-(1 / 2 \sqrt{3} Q)]$. For the parameters used in Fig. $1(\mathrm{~b}),\left|\Gamma\left(\omega_{0}\right)\right| / \omega_{c}^{2}=1.0$, so that the system is intermediate between the weak and strong focusing regimes. Figure $1(\mathrm{c})$ describes the strong focusing regime, with $\left|\Gamma\left(\omega_{0}\right)\right| / \omega_{c}^{2}=0.01$, in which solenoidal transport gives twice as much growth as quadrupole transport for the same value of $\omega_{c}$, and BBU growth is largest at $\omega_{\max }=\omega_{0}$.

In actual accelerating cavities, the normally degenerate $\mathrm{TM}_{110}$ modes may be split because of perturbations to cylindrical symmetry from coupling loops, damping, slots, drive rods, etc., ${ }^{2,13}$ We can model the case where the perturbed modes have vertical and horizontal polarizations by using separate resonant frequencies $\left(\omega_{x}\right.$ and $\left.\omega_{y}\right)$ and quality factors $\left(Q_{x}\right.$ and $Q_{y}$ ) for the linearly polarized modes in Equations (14a) and (14b).

For a disturbance of the form $e^{i \omega t-i k z}$, Eqs. (13) and (14) then yield the dispersion relation

$$
\Omega^{4}+\Omega^{2}\left(-\omega_{c}^{2}+\Gamma_{x}+\Gamma_{y}\right)+\Gamma_{x} \Gamma_{y}=0,
$$

where $\Omega=\omega-k v, \quad \Gamma_{x}=2 \omega_{x}^{4} \epsilon /\left(-\omega^{2}+\omega_{x}^{2}\right.$ $\left.+i \omega \omega_{x} / Q\right)$, and $\Gamma_{y}=2 \omega_{y}^{4} \epsilon /\left(-\omega^{2}+\omega_{y}^{2}+i \omega \omega_{y} / Q\right)$. Note that $\Gamma_{x}$ is a function of $\omega$ whose magnitude is maximum for $\omega \cong \omega_{x}$, while the magnitude of $\Gamma_{y}$ is maximum for $\omega \cong \omega_{y}$.

In the weak focusing regime where $\left|\Gamma_{x}\left(\omega_{x}\right)\right|$, $\left|\Gamma_{y}\left(\omega_{y}\right)\right|>\omega_{c}^{2}$, Eq. (18) has solutions obeying $\Omega^{2}$ $+\Gamma_{x}=0$ for $\omega \cong \omega_{x}$, and $\Omega^{2}+\Gamma_{y}=0$ for $\omega \cong \omega_{y}$. These results are identical to the one-dimensional result of Eq. (7).

In the strong focusing regime, $\left|\Gamma_{x}\left(\omega_{x}\right)\right|,\left|\Gamma_{y}\left(\omega_{y}\right)\right|$ $<\omega_{c}^{2}$, the dispersion relation (18) becomes

$$
\begin{aligned}
& \Omega= \pm\left\{\omega_{c}-\left[\left(\Gamma_{x}+\Gamma_{y}\right) / 2 \omega_{c}\right]\right\} \\
& \Omega= \pm\left(\Gamma_{x} \Gamma_{y}\right)^{1 / 2} / \omega_{c} .
\end{aligned}
$$

If the $\mathbf{T M}_{110}$ modes have sufficiently different frequencies so that $\left|\omega_{x}-\omega_{y}\right|>\omega_{x} / Q_{x}, \omega_{y} / Q_{y}$, then Eqs. (19) and (20) are no longer approximated by Eqs. (16) and (17). A maximum in growth occurs for $\omega=\omega_{x}$, where Eq. (19) becomes $\Omega= \pm\left[\omega_{c}-\Gamma_{x}\left(\omega_{x}\right) / 2 \omega_{c}\right]$. Similarly, a maximum occurs for $\omega=\omega_{y}$, where Eq. (19) becomes $\Omega$ $= \pm\left[\omega_{c}-\Gamma_{y}\left(\omega_{y}\right) / 2 \omega_{c}\right]$. These are identical to the results of the one-dimensional model. The growing mode of Eq. (20) has greatly reduced growth compared with Eq. (19).

In the strong focusing regime, a solenoidal focusing system with cylindrical pillbox cavities will have twice the BBU growth rate of a quadrupole focusing system with the same betatron wavelength. The solenoidal focusing growth rate may be halved by perturbing the cavities so that vertical and horizontal linear polarizations of the $\mathrm{TM}_{110}$ mode have sufficiently different resonant frequencies. However, if the perturbed cavities are oriented so that the linearly po- 
larized modes of identical resonant frequency are aligned in the helical Larmor frame, BBU growth is not reduced by the perturbation.

The preceding equations treat the beam current as constant, and the transverse impulsive forces from the accelerating cavities as a continuous force per unit length. These approximations limit the dispersion relations to the cases where instability scale lengths ( $e$ folding length, wavelength) are long compared to the cavity spacing, and the beam current is constant.

\section{DISCRETE SPATIAL EFFECTS}

\section{A. One-dimensional model}

If the betatron wavelength or BBU $e$ folding length do not greatly exceed the spacing $L$ of the accelerator cavities, we can no longer treat the transverse impulsive forces from the cavities as a continuous force per unit length. We consider a quadrupole focusing system with cavities of length $l$ and separation $L$, with $l<L$. Treating the forces from the cavities as impulses, we have

$$
\begin{aligned}
& \frac{d}{d t} \gamma \frac{d x}{d t}+\gamma \omega_{c}^{2} x=a L \sum_{j=-\infty}^{\infty} \delta(z-j L), \\
& \left(\frac{\partial^{2}}{\partial t^{2}}+\frac{\omega_{0}}{Q} \frac{\partial}{\partial t}+\omega_{0}^{2}\right) a=2 \gamma \omega_{0}^{4} \in x .
\end{aligned}
$$

These equations are invariant under space translation by distance $L$ and arbitrary time translations. Thus, we consider solutions of the form $x=x_{0}(z) e^{i \omega t}$, where $x_{0}(z)$ $=u(z) e^{-i k z}$, and $u(z)$ is periodic with period $L$. Because $e^{-i k L}=e^{-i L(k+2 m \pi / L)}$ for integral values of $m, k$ and $k$ $+2 m \pi / L$ describe the same solution for any given $\omega$. As a result, $\Omega$ and $\Omega-2 m \pi v / L$ describe the same solution for any given $\omega$. Inserting the solution form, we obtain

$\left[\left(i \omega+v \frac{d}{d z}\right)^{2}+\omega_{c}^{2}\right] x_{0}=\Gamma x_{0} L \sum_{j=-\infty}^{\infty} \delta(z-j L)$.

Because of the periodicity of $u(z)$, we need only solve Eq. (23) for $0<z<L$. In this region, the right-hand side of Eq. (23) vanishes, yielding the solution

$$
\begin{aligned}
x_{0}(z)= & A \exp \left(\frac{i z}{v}\left(-\omega+\omega_{c}\right)\right) \\
& +B \exp \left(\frac{i z}{v}\left(-\omega-\omega_{c}\right)\right) .
\end{aligned}
$$

Continuity at $z=0$ implies

$$
x_{0}(0+)=x_{0}(0-)=e^{i k L} x_{0}(L-),
$$

while Eq. (23) implies the jump condition:

$$
\frac{d x_{0}}{d z}(0+)-\frac{d x_{0}}{d z}(0-)=\frac{\Gamma L}{v^{2}} x_{0}(0),
$$

where

$$
\frac{d x_{0}}{d z}(0-)=e^{i k L} \frac{d x_{0}}{d z}(L-) .
$$

Inserting the ansatz (24) into Eqs. (25) and (26) yields two equations in three unknowns: $A, B$, and $k$. Eliminating $A$ and $B$ gives the dispersion relation

$$
\cos \left(\frac{L \Omega}{v}\right)=\cos \frac{L \omega_{c}}{v}+\frac{\Gamma L}{2 \omega_{c} v} \sin \frac{L \omega_{c}}{v},
$$

where $\Omega=\omega-k v$. In the limit that the betatron wavelength and $\mathrm{BBU} e$ folding length are long compared with the cavity spacing, this equation reduces to the continuum dispersion relation of Eq. (3).

Equation (27a) may also be written as ${ }^{14}$

$$
\begin{gathered}
\left(-\omega^{2}+\omega_{0}^{2}+\frac{i \omega \omega_{0}}{Q}\right)\left(\cos \frac{L \Omega}{v}-\cos \frac{L \omega_{c}}{v}\right) \\
=\frac{\omega_{0}^{4} \epsilon L}{\omega_{c} v} \sin \frac{L \omega_{c}}{v} .
\end{gathered}
$$

This form makes explicit the coupling between the cavity mode and the beam mode. For $k L=2 \pi n$ and complex $\omega$, Eq. (27) gives the dispersion relation for a recirculating induction accelerator with one cavity, quadrupole focusing, and beam pulse length equal to the recirculation time. ${ }^{15}$

For $L=(n / 2) \lambda_{b}$, where $n$ is an integer and $\lambda_{b}$ $=2 \pi v / \omega_{c}$ is the betatron wavelength, the dispersion relation becomes $\Omega= \pm \omega_{c}$. Thus, there is no BBU growth for any value of $\omega$ when the cavity spacing $L$ equals $(n / 2) \lambda_{b}$. Intuitively, this is a reasonable result: An impulse in the $x$ direction provided by an accelerator cavity will not result in a deflection in the $x$ direction in the subsequent cavity if the spacing is $(n / 2) \lambda_{b}$, thwarting the mechanism of BBU growth. A finite level of BBU growth will result if the cavity spacing is not exactly equal to $(n / 2) \lambda_{b}$ and because of the finite cavity length $l$.

In the strong focusing regime with $\left|\Gamma\left(\omega_{0}\right)\right|<\omega_{c}^{2}$, Eq. (27) is approximated by the one-dimensional continuum model dispersion relation of Eq. (10) for cavity spacings sufficiently far from $(n / 2) \lambda_{b}$ that

$$
\left|L-(n / 2) \lambda_{b}\right| / L>\left|\Gamma\left(\omega_{0}\right)\right| / 2 \omega_{c}^{2}
$$

\section{B. Two-dimensional model}

For solenoidal focusing, a two-dimensional model can account for the discrete spatial effects when the cavity spacing $L$ is not small compared to the betatron or BBU $e$ folding length. We consider cylindrical pillbox cavities:

$$
\begin{aligned}
& \frac{d}{d t} \gamma \frac{d x}{d t}+\gamma \omega_{c} \frac{d y}{d t}=a_{x} L \sum_{j=-\infty}^{\infty} \delta(z-j L), \\
& \frac{d}{d t} \gamma \frac{d y}{d t}-\gamma \omega_{c} \frac{d x}{d t}=a_{y} L \sum_{j=-\infty}^{\infty} \delta(z-j L), \\
& \left(\frac{\partial^{2}}{\partial t^{2}}+\frac{\omega_{0}}{Q} \frac{\partial}{\partial t}+\omega_{0}^{2}\right) a_{x}=2 \gamma \omega_{0}^{4} \epsilon x, \\
& \left(\frac{\partial^{2}}{\partial t^{2}}+\frac{\omega_{0}}{Q} \frac{\partial}{\partial t}+\omega_{0}^{2}\right) a_{y}=2 \gamma \omega_{0}^{4} \epsilon y .
\end{aligned}
$$


These equations are invariant under space translation by distance $L$ and arbitrary time translations. Thus, we consider solutions of the form $x=x_{0}(z) e^{i \omega t}$, where $x_{0}(z)$ $=u(z) e^{-i k z}$, and $u(z)$ is periodic with period $L$; and similarly for $y$. We thereby obtain

$$
\begin{gathered}
\left(i \omega+v \frac{d}{d z}\right)^{2} x_{0}+\omega_{c}\left(i \omega+v \frac{d}{d z}\right) y_{0} \\
=\Gamma x_{0} L \sum_{j=-\infty}^{\infty} \delta(z-j L), \\
\left(i \omega+v \frac{d}{d z}\right)^{2} y_{0}-\omega_{c}\left(i \omega+v \frac{d}{d z}\right) x_{0} \\
=\Gamma y_{0} L \sum_{j=-\infty}^{\infty} \delta(z-j L) .
\end{gathered}
$$

In the region $0<z<L$, the right-hand side of Eqs. (30) vanish, yielding the solution

$$
\begin{aligned}
x_{0}(z)= & A \exp \left(\frac{i z}{v}\left(-\omega+\omega_{c}\right)\right) \\
& +B \exp \left(\frac{i z}{v}\left(-\omega-\omega_{c}\right)\right) \\
& +C \exp \left(\frac{-i \omega z}{v}\right), \\
y_{0}(z)= & -i A \exp \left(\frac{i z}{v}\left(-\omega+\omega_{c}\right)\right) \\
& +i B \exp \left(\frac{i z}{v}\left(-\omega-\omega_{c}\right)\right) \\
& -i D \exp \left(\frac{-i \omega z}{v}\right) .
\end{aligned}
$$

Continuity at $z=0$ implies

$$
\begin{aligned}
& x_{0}(0+)=x_{0}(0-)=e^{i k L} x_{0}(L-), \\
& y_{0}(0+)=y_{0}(0-)=e^{i k L} y_{0}(L-),
\end{aligned}
$$

while Eqs. (30) imply the jump conditions

$$
\begin{aligned}
& \frac{d x_{0}}{d z}(0+)-\frac{d x_{0}}{d z}(0-)=\frac{\Gamma L}{v^{2}} x_{0}(0), \\
& \frac{d y_{0}}{d z}(0+)-\frac{d y_{0}}{d z}(0-)=\frac{\Gamma L}{v^{2}} y_{0}(0),
\end{aligned}
$$

where

$$
\frac{d x_{0}}{d z}(0-)=e^{i k L} \frac{d x_{0}}{d z}(L-)
$$

and

$$
\frac{d y_{0}}{d z}(0-)=e^{i k L} \frac{d y_{0}}{d z}(L-) .
$$

Inserting the ansatz (31) into Eqs. (32) and (33) yields four equations in five unknowns: $A, B, C, D$, and $k$. Eliminating $A, B, C$, and $D$ gives the dispersion relation

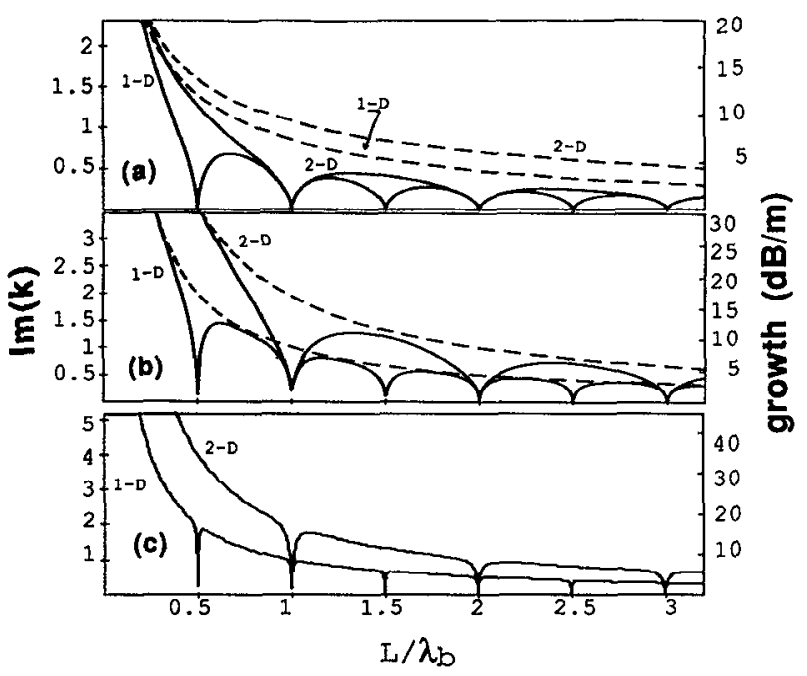

FIG. 2. Graphs of the spatial growth rate $\operatorname{Im}(k)$ as a function of the normalized cavity spacing $L / \lambda_{b}$. Dashed lines show the growth rates for the continuum models. Growth is shown at an angular frequency $\omega$ for which growth is near maximum. The parameters used are kinetic energy $=700 \mathrm{keV}$, current $=100 \mathrm{~A}, \mathrm{TM}_{110}$ mode frequency $=2.5 \mathrm{GHz}, Q$ $=200$, and $l=1 \mathrm{~cm}$. (a) Betatron wavelength $=5 \mathrm{~m} ; \omega=\omega_{0}[1$ - $(1 / 2 \sqrt{3 Q})$ ). (b) Betatron wavelength $=0.5 \mathrm{~m} ; \omega=\omega_{0}$. (c) Betatron wavelength $=5 \mathrm{~cm} ; \omega=\omega_{0}$.

$$
\cos \left(\frac{L \Omega}{v} \pm \frac{L \omega_{c}}{2 v}\right)=\cos \frac{L \omega_{c}}{2 v}+\frac{\Gamma L}{\omega_{c} v} \sin \frac{L \omega_{c}}{2 v}
$$

where $\Omega=\omega-k v$. Because Eqs. (28) and (29) are separable in the helical Larmor frame, Eq. (34) may also be obtained from Eq. (27a) by letting $\omega_{c} \rightarrow \omega_{c} / 2$ and $\Omega$ $\rightarrow \Omega \pm \omega_{c} / 2$.

Four modes are described by Eq. (34). In the limit that the betatron wavelength and $\mathrm{BBU} e$ folding length are long compared with the cavity spacing, this equation reduces to the two-dimensional continuum dispersion relation of Eq. (15).

For $L=n \lambda_{b}$, where $n$ is an integer, the dispersion relation becomes $\Omega=0, \pm \omega_{c}$. Thus, there is no BBU growth for any value of $\omega$ when the cavity spacing $L$ equals $n \lambda_{b}$, where $\lambda_{b}$ is the betatron wavelength. Intuitively, this is a reasonable result: An impulse provided by an accelerator cavity will not result in a deflection in the subsequent cavity if the spacing is $n \lambda_{b}$, thwarting the mechanism of BBU growth. Unlike the one-dimensional model applicable to quadrupole focusing, a spacing of $(n / 2) \lambda_{b}$, with odd integer $n$, will no longer suppress BBU growth. This is a result of $x-y$ coupling. An impulse in the $x$ direction will result in a deflection in the $y$ direction in the subsequent cavity with a spacing of $(n / 2) \lambda_{b}$ with odd integer $n$, so that the BBU instability can grow.

In the strong focusing regime, where $\left|\Gamma\left(\omega_{0}\right)\right|<\omega_{c}^{2}$, Eq. (34) is approximated by the two-dimensional continuum dispersion relations of Eqs. (16) and (17) for cavity spacings sufficiently far from $n \lambda_{b}$ that $\left|L-n \lambda_{b}\right| / L$ $>2\left|\Gamma\left(\omega_{0}\right)\right| / \omega_{c}^{2}$

In Fig. 2, we display the BBU growth rate as a function of the normalized cavity spacing, $L / \lambda_{b}$, for the one- 
and two-dimensional models that account for finite cavity spacing by treating cavity forces as impulses [Eqs. (27) and (34)]. The corresponding results from the continuum models [Eqs. (3) and (15)] are shown for comparison. In Fig. 2(a), the system is intermediate between the weak and strong focusing regimes; growth is significantly reduced by the finite cavity spacing, for the values of $L / \lambda_{b}$ depicted. In Figs. 2(b) and 2(c), the systems are in the strong focusing regime. Finite cavity spacing reduces growth for $L / \lambda_{b}$ $\cong n / 2$ for the one-dimensional model, and $L / \lambda_{b} \cong n$ for the two-dimensional model, where $n$ is an integer. As expected, the growth of the one-dimensional model is approximately equal to that predicted by the 1D continuum model for cavity spacings sufficiently different from $(n / 2) \lambda_{b}$ that

$$
\left|L-(n / 2) \lambda_{b}\right| / L>\left|\Gamma\left(\omega_{0}\right)\right| / 2 \omega_{c}^{2} .
$$

For the two-dimensional model, growth is approximated by the $2 \mathrm{D}$ continuum model for $\left|L-n \lambda_{b}\right| / L$ $>2\left|\Gamma\left(\omega_{0}\right)\right| / \omega_{c}^{2}$.

We finally consider the case where the linearly polarized ' $\mathbf{T M}_{110}$ modes that deflect in the $x$ and $y$ directions are perturbed so that their resonant frequencies are sufficiently different that $\left|\omega_{x}-\omega_{y}\right|>\omega_{x} / Q_{x}, \omega_{y} / Q_{y}$. Then, for $\omega$ $\cong \omega_{x}$, Eq. (30) approximately holds with $\Gamma=\Gamma_{x}$ on the right-hand side of Eq. (30a), and the right-hand side of Eq. (30b) set to zero. For a centered beam, Eq. (30b) can then be used to transform Eq. (30a) into the one-dimensional result of Eq. (23). Consequently, the one-dimensional dispersion relation of Eq. (27) will apply for $\omega$ $\cong \omega_{x}$. Because this is an approximate result, the BBU growth for $L=(n / 2) \lambda_{b}$ may not completely vanish. As in the continuum models, when the two lincar polarizations of the $\mathrm{TM}_{110}$ mode are perturbed so that their frequency response curves do not greatly overlap, the onedimensional dispersion relation applies.

\section{c. Finite cavity length}

The preceding calculations have shown that BBU growth vanishes with the proper choice of cavity spacing, if the forces from the cavities are modeled as impulses. We now consider the finite growth that will occur for nonzero cavity length $l$ and errors in cavity spacing from an integral multiple of the half-betatron wavelength. We restrict the discussion to the one-dimensional model appropriate for quadrupole focusing. By letting $\omega_{c} \rightarrow \omega_{c} / 2$ and $\Omega \rightarrow \Omega$ $\pm \omega_{c} / 2$, the dispersion relation we obtain will also be applicable to solenoidal focusing with cylindrically symmetric cavities.

For a cavity spacing $L=n \pi v / \omega_{c}+\delta$, where $\delta$ is the error in spacing from an integral multiple of the half-betatron wavelength, Eq. (27) gives the result for cavity forces modeled as impulses:

$$
\Omega= \pm \omega_{c} \pm[-(\Gamma \delta / L)]^{1 / 2} .
$$

Equation (35) is approximately true for $|\delta|<\lambda_{b} / 2 \pi$, where $\lambda_{b}=2 \pi v / \omega_{c}$ is the betatron wavelength. In addition, $|\Gamma \delta / L|^{1 / 2}<\omega_{d} / 2 \pi$ must be obeyed. Additional values for $\Omega$ differing by $2 m \pi v / L$, where $m$ is any integer, describe the same solution.
To model finite cavity lengths, we begin with the equations for finite cavity separation, replacing the $\delta$ function representing the impulsive acceleration in the cavity with a flattop function. This approach should be valid for small cavity lengths for which the electron transit time is small compared to the $\mathrm{TM}_{110}$ period. Thus, we have

$$
\begin{aligned}
& \frac{d}{d t} \gamma \frac{d x}{d t}+\gamma \omega_{c}^{2} x=a L \sum_{j=-\infty}^{\infty} \delta_{l}(z-j L), \\
& \left(\frac{\partial^{2}}{\partial t^{2}}+\frac{\omega_{0}}{Q} \frac{\partial}{\partial t}+\omega_{0}^{2}\right) a=2 \gamma \omega_{0}^{4} \in x .
\end{aligned}
$$

Here, the function $\delta_{l}(z)=1 / l$ for $-l<z<0$ and zero elsewhere. These equations are invariant under space translation by distance $L$ and arbitrary time translations, so we consider solutions of the form $x=x_{0}(z) e^{i \omega t}$, where $x_{0}(z)=u(z) e^{-i k z}$, and $u(z)$ is periodic with period $L$. We thereby obtain

$$
\left[\left(i \omega+v \frac{d}{d z}\right)^{2}+\omega_{c}^{2}\right] x_{0}=\Gamma x_{0} L \sum_{j--\infty}^{\infty} \delta_{l}(z-j L) .
$$

Because of the periodicity of $u(z)$, we need only solve Eq. (38) for $-l<z<L-l$. In the region $0<z<L-l$, the right-hand side of Eq. (38) vanishes, yielding the solution

$$
\begin{aligned}
x_{0}(z)= & A \exp \left(\frac{i z}{v}\left(-\omega+\omega_{c}\right)\right) \\
& +B \exp \left(\frac{i z}{v}\left(-\omega-\omega_{c}\right)\right) .
\end{aligned}
$$

For $-l<z<0$, we have

$$
\begin{aligned}
x_{0}(z)= & C \exp \left\{\frac{i z}{v}\left[-\omega+\left(\omega_{c}^{2}-\frac{\Gamma L}{l}\right)^{1 / 2}\right]\right\} \\
& +D \exp \left\{\frac{i z}{v}\left[-\omega-\left(\omega_{c}^{2}-\frac{\Gamma L}{l}\right)^{1 / 2}\right]\right\} .
\end{aligned}
$$

Continuity of $x_{0}$ implies that $x_{0}(0+)=x_{0}(0-)$ and

$$
x_{0}(-l+)=x_{0}(-l-)=e^{i k L} x_{0}(L-l-) \text {. }
$$

Continuity of $d x_{0} / d z$ at $z=-l$ and $z=0$ gives two analogous equations, so we have four equations in the unknowns $A, B, C, D$, and $k$. Eliminating $A, B, C$, and $D$, we have the equation

$$
\begin{aligned}
0= & (M-P)(R-S)\left(e^{(P+M) L}(P+M) l\right. \\
& \left.+e^{-2 i k L-(P+M) l}\right)-(R-P)^{2}\left(e^{(P-i k) L-(P+R) l}\right. \\
& \left.+e^{(M-i k) L-(M+S) l}\right)+(S-P)^{2}\left(e^{(P-i k) L-(P+S) l}\right. \\
& \left.+e^{(M-i k) L-(M+R) l}\right)
\end{aligned}
$$

where $P=(i / v)\left(-\omega+\omega_{c}\right), M=(i / v)(-\omega$ $\left.-\omega_{c}\right), R=(i / v)\left[-\omega+\left(\omega_{c}^{2}-\Gamma L / l\right)^{1 / 2}\right]$, and $S=(i / v)\left[-\omega-\left(\omega_{c}^{2}-\Gamma L / l\right)^{1 / 2}\right]$.

Writing Eq. (41) in terms of $\Omega=\omega-v k, \omega_{c}$, and $\theta=\left(\omega_{c}^{2}-\Gamma L / l\right)^{1 / 2}$, we obtain a quadratic equation in $e^{i L \Omega / v}$, yielding the dispersion relation

$$
\cos (L \Omega / v)=\alpha,
$$


where

$$
\begin{aligned}
\alpha= & -\frac{1}{4 \omega_{c} \theta}\left[\left(\omega_{c}-\theta\right)^{2} \cos \left(\frac{\omega_{c} L}{v}-\left(\omega_{c}+\theta\right) \frac{l}{v}\right)\right. \\
& \left.-\left(\omega_{c}+\theta\right)^{2} \cos \left(\frac{\omega_{c} L}{v}-\left(\omega_{c}-\theta\right) \frac{l}{v}\right)\right] .
\end{aligned}
$$

If $\alpha$ is expanded to first order in $l$, Eq. (42) reduces to Eq. (27), where cavity forces are modeled as impulses. For cavity spacings close to an integral multiple of the halfbetatron wavelength, $L=n \pi v / \omega_{c}+\delta$, where $\delta$ is the error in spacing. We expand Eq. (43) through fourth order in $l$ and $\delta$, noting that $\Gamma \propto \epsilon \propto l$, thereby obtaining the approximate dispersion relation

$$
\begin{aligned}
\Omega= & \pm \omega_{c}\left(1-\frac{\delta}{L}\right) \\
& \pm\left[-\frac{l \delta}{L^{2}}\left(\frac{\Gamma L}{l}\right)+\frac{\delta^{2}}{L^{2}} \omega_{c}^{2}\right. \\
& +\frac{l^{4}}{L^{4}} \frac{n^{2} \pi^{2}}{12 \omega_{c}^{2}}\left(\frac{\Gamma L}{l}\right)^{2}-\frac{l^{3} \delta}{L^{4}} \frac{n^{2} \pi^{2}}{6 \omega_{c}^{2}}\left(\frac{\Gamma L}{l}\right)^{2} \\
& \left.+\frac{l \delta^{3}}{L^{4}} \frac{n^{2} \pi^{2}}{6}\left(\frac{\Gamma L}{l}\right)-\frac{\delta^{4}}{L^{4}} \frac{n^{2} \pi^{2} \omega_{c}^{2}}{12}\right]^{1 / 2} .
\end{aligned}
$$

The expression $(\Gamma L / l)$ has no dependence upon $l$. In order for Eq. (44) to be valid, it is necessary that $|\delta|, l$ $<\lambda_{b} / 2 \pi$, and that the terms on the right-hand side containing $\delta$ and $l$ be small compared to $\omega_{c} / n \pi$. We note that the lowest-order terms reproduce Eq. (35), where the forces from the cavities are modeled as impulses. For zero spacing error $(\delta=0)$, Eq. (44) becomes

$$
\Omega= \pm \omega_{c} \pm \frac{l^{2}}{L^{2}} \frac{n \pi}{2 \sqrt{3} \omega_{c}}\left(\frac{\Gamma L}{l}\right)
$$

so that growth does not vanish for $L=(n / 2) \lambda_{b}$ when finite cavity length is considered. Comparing Eqs. (35) and (45) in the strong focusing regime $\left(|\Gamma|<\omega_{c}^{2}\right)$, we note that the BBU growth from a spacing error $\delta$ will exceed the growth from a cavity length $l$ when $|\delta| \sim l$, unless $n$ is very large. Thus, a spacing error will usually lead to greater BBU growth than a cavity length of comparable magnitude.

Comparing Eq. (45) with the continuum model dispersion relation in the strong focusing regime, Eq. (10), we note that BBU growth is modified by the factor $(2 \pi / \sqrt{3})$ $\times\left(l / \lambda_{b}\right)$ when the cavity spacing is $(n / 2) \lambda_{b}$. The reduction in BBU growth is substantial when $\left(l / \lambda_{b}\right)<1$.

As an illustration of Eq. (45), we calculate the nonzero BBU growth resulting from the finite cavity length for quadrupole transport with $L=\lambda_{b} / 2$, for the parameters of Fig. 2. For the parameters of Fig. 2(a), a cavity length of $l=1 \mathrm{~cm}$ gives $\operatorname{Im}(k)=0.015 \mathrm{~m}^{-1}$, corresponding to a growth rate of $0.13 \mathrm{~dB} / \mathrm{m}$. For the case of Fig. 2(b), a growth of $1.3 \mathrm{~dB} / \mathrm{m}$ is obtained. For the parameters of Fig. 2 (c), a growth of $13 \mathrm{~dB} / \mathrm{m}$ is given by Eq. (45). Because $l / \lambda_{b}$ is not small compared to 1 , the growth is not significantly reduced compared with the continuum model.
Moreover, the requirement that $l<\lambda_{b} / 2 \pi$ is not obeyed for Fig. 2(c), so that $13 \mathrm{~dB} / \mathrm{m}$ is not an accurate value.

\section{PERIODICALLY PULSED BEAM}

\section{A. One-dimensional model}

In this section, we consider a periodically pulsed beam, which can represent a micropulse train, in the limit of vanishing pulselength. The beam current is modeled as a train of $\delta$ functions separated by time $\tau$. We assume that the betatron wavelength and BBU $e$ folding length are long compared with the cavity separation, so that the impulsive transverse forces can be modeled as a continuous force per unit length. For a quadrupole focusing system, we have

$$
\begin{aligned}
& \frac{d}{d t} \gamma \frac{d x}{d t}+\gamma \omega_{c}^{2} x=a \\
& \left(\frac{\partial^{2}}{\partial t^{2}}+\frac{\omega_{0}}{Q} \frac{\partial}{\partial t}+\omega_{0}^{2}\right) a \\
& \quad=2 \gamma \omega_{0}^{4} \epsilon x \tau \sum_{j=-\infty}^{\infty} \delta\left[t-\left(j \tau+\frac{z}{v}\right)\right] .
\end{aligned}
$$

The quantity $\epsilon$ is defined by Eq. (2) using the average beam current. Defining the variables $T=t-z / v$ and $Z$ $=z$, we can write

$v^{2} \gamma \frac{\partial^{2} x}{\partial Z^{2}}+\gamma \omega_{c}^{2} x=a$,

$$
\left(\frac{\partial^{2}}{\partial T^{2}}+\frac{\omega_{0}}{Q} \frac{\partial}{\partial T}+\omega_{0}^{2}\right) a=2 \gamma \omega_{0}^{4} \epsilon x \tau \sum_{j=-\infty}^{\infty} \delta(T-j \tau)
$$

This system is invariant under arbitrary translations in $Z$, and periodic in $T$ with period $\tau$. We thus consider solutions of the form $a=a_{0}(T) e^{i \Omega Z / v}$ where $a_{0}(T)=u(T) e^{i \omega T}$, and $u(T)$ is periodic with period $\tau$. In terms of the variables $t$ and $z, a=u(t-z / v) e^{-i k z+i \omega t}$, where $\Omega=\omega$ $-k v$. Because $e^{i \omega \tau}=e^{i \tau(\omega+2 m \pi / \tau)}$ for integral $m, \omega$, and $\omega+2 m \pi / \tau$ describe the same solution, giving the same value of $\Omega$. Substituting the desired solution form into Eqs. (48) and (49) yields

$$
\left(\frac{d^{2}}{d T^{2}}+\frac{\omega_{0}}{Q} \frac{d}{d T}+\omega_{0}^{2}\right) a_{0}=\frac{2 \omega_{0}^{4} \epsilon \tau a_{0}}{\omega_{c}^{2}-\Omega^{2}} \sum_{j=-\infty}^{\infty} \delta(T-j \tau) .
$$

In the region $0<T<\tau$, the right-hand side of Eq. (50) vanishes, yielding the solution

$$
\begin{aligned}
a_{0}= & A \exp \left\{\left[i \omega_{0} \sqrt{1-\left(1 / 4 Q^{2}\right)}-\left(\omega_{0} / 2 Q\right)\right] T\right\} \\
& +B \exp \left\{\left[-i \omega_{0} \sqrt{1-\left(1 / 4 Q^{2}\right)}\right.\right. \\
& \left.\left.-\left(\omega_{0} / 2 Q\right)\right] T\right\} .
\end{aligned}
$$

Continuity at $T=0$ implies 


$$
a_{0}(0+)=a_{0}(0-)=e^{-i \omega \tau} a_{0}(\tau-),
$$

while Eq. (50) implies the jump condition:

$$
\frac{d a_{0}}{d T}(0+)-\frac{d a_{0}}{d T}(0-)=\frac{2 \omega_{0}^{4} \epsilon \tau a_{0}(0)}{\omega_{c}^{2}-\Omega^{2}},
$$

where

$$
\frac{d a_{0}}{d T}(0-)=e^{-i \omega \tau} \frac{d a_{0}}{d T}(\tau-) .
$$

Inserting the ansatz (51) into Eqs. (52) and (53) yields two equations in three unknowns: $A, B$, and $\Omega$. Eliminating $A$ and $B$ gives the dispersion relation

$\Omega^{2}-\omega_{c}^{2}=\frac{\omega_{0}^{3} \epsilon \tau \sin \left[\omega_{0} \sqrt{1-\left(1 / 4 Q^{2}\right)} \tau\right]}{2 \sqrt{1-\left(1 / 4 Q^{2}\right)} \sin \left\{\left[\omega_{0} \sqrt{1-\left(1 / 4 Q^{2}\right)}-\omega+\left(i \omega_{0} / 2 Q\right)\right](\tau / 2)\right\} \sin \left\{\left[-\omega_{0} \sqrt{1-\left(1 / 4 Q^{2}\right)}-\omega+\left(i \omega_{0} / 2 Q\right)\right](\tau / 2)\right\}}$.

We note that $\omega$ and $\omega+2 m \pi / \tau$ give the same value of $\Omega$, as expected. If the period between bunches $\tau$ is sufficiently small that $\omega_{0} \tau<1$, and we consider frequencies $\omega$ sufficiently small that $\omega \tau<1$, then the right-hand side of Eq. (54) reduces to $-\Gamma$, giving the one-dimensional continuum dispersion relation of Eq. (3). A BBU disturbance that does not oscillate in time is described by Eq. (54) with $\omega=0$. Consequently, the growth rate given by Eq. (54) for $\omega=0$ agrees with that obtained by other authors for a steady-state disturbance resulting from a misaligned beam ${ }^{16,17}$ [see Eq. (47) of Ref. 16 and Eq. (16) of Ref. 17]. The growth rate given by Eq. (54) for $\omega_{c}=0$ has also been described in Ref. 6.

We note that the right-hand side of Eq. (54) vanishes for $\tau=(n / 2) \tau_{0}$, where $\tau_{0}=2 \pi /\left(\omega_{0} \sqrt{1-1 / 4 Q^{2}}\right)$ is the period of the $\mathrm{TM}_{110}$ cavity mode. Thus, BBU growth vanishes when the bunch separation is an integral number of half-periods of the $\mathbf{T M}_{110}$ mode. ${ }^{18}$

For frequencies near $\omega_{0}$, obeying $\left|\omega-\omega_{0}\right|$ $\lesssim \omega_{0} / 2 Q$, and bunch separations sufficiently different from $(n / 2) \tau_{0}$, obeying $\left|\tau-(n / 2) \tau_{0}\right|>\tau / 2 Q$, and $n<Q$, the right-hand side of Eq. (54) reduces to $-\Gamma$, so that the one-dimensional continuum dispersion relation applies. Because $\left|\omega_{\max }-\omega_{0}\right| \lesssim \omega_{0} / 2 Q$, BBU growth is given by the $1 D$ continuum theory for $\left|\tau-(n / 2) \tau_{0}\right|>\tau / 2 Q$ and $n<Q$.

\section{B. TWO-DIMENSIONAL MODEL}

For a periodically pulsed beam in a solenoidal focusing system with cylindrical pillbox cavities, we consider a twodimensional model. Again, we assume that the betatron wavelength and $\mathrm{BBU} e$ folding length are long compared with the cavity separation so that a continuum description can be used for the acceleration of the beam by the cavities. In terms of the variables $T=t-z / v$ and $Z=z$, we have

$v^{2} \gamma \frac{\partial^{2} x}{\partial Z^{2}}+\gamma \omega_{c} v \frac{\partial y}{\partial Z}=a_{x}$

$v^{2} \gamma \frac{\partial^{2} y}{\partial Z^{2}}-\gamma \omega_{c} v \frac{\partial x}{\partial Z}=a_{y}$ $\left(\frac{\partial^{2}}{\partial T^{2}}+\frac{\omega_{0}}{Q} \frac{\partial}{\partial T}+\omega_{0}^{2}\right) a_{x}=2 \gamma \omega_{0}^{4} \epsilon x \tau \sum_{j=-\infty}^{\infty} \delta(T-j \tau)$,

$\left(\frac{\partial^{2}}{\partial T^{2}}+\frac{\omega_{0}}{Q} \frac{\partial}{\partial T} \mid \omega_{0}^{2}\right) a_{y}=2 \gamma \omega_{0}^{4} \epsilon y \tau \sum_{j=-\infty}^{\infty} \delta(T-j \tau)$

This system is invariant under arbitrary translations in $Z$, and periodic in $T$ with period $\tau$. We thus consider solutions of the form $a_{x}=a_{x 0}(T) e^{i \Omega Z / v}$ where $a_{x 0}(T)$ $=u(T) e^{i \omega T}$, and $u(T)$ is periodic with period $\tau$. A similar form is assumed for $a_{y}, x$, and $y$. In terms of the variables $t$ and $z, a_{x}=u(t-z / v) e^{-i k z+i \omega t}$, where $\Omega=\omega-k v$. Substituting this solution form into Eqs. (55) and (56) yields

$$
\begin{aligned}
& \left(\frac{d^{2}}{d T^{2}}+\frac{\omega_{0}}{Q} \frac{d}{d T}+\omega_{0}^{2}\right) a_{x 0} \\
& \quad=\frac{2 \omega_{0}^{4} \epsilon \tau\left(a_{x 0}+i \omega_{c} a_{y 0} / \Omega\right)}{\omega_{c}^{2}-\Omega^{2}} \sum_{j--\infty}^{\infty} \delta(T-j \tau),
\end{aligned}
$$

$$
\begin{aligned}
& \left(\frac{d^{2}}{d T^{2}}+\frac{\omega_{0}}{Q} \frac{d}{d T}+\omega_{0}^{2}\right) a_{\not 0} \\
& \quad=\frac{2 \omega_{0}^{4} \epsilon \tau\left(a_{y 0}-i \omega_{c} a_{x 0} / \Omega\right)}{\omega_{c}^{2}-\Omega^{2}} \sum_{j=-\infty}^{\infty} \delta(T-j \tau) .
\end{aligned}
$$

In the region $0<T<\tau$, the right-hand side of Eqs. (57) vanishes, yielding the solution

$$
\begin{aligned}
a_{x 0}= & A \exp \left\{\left[i \omega_{0}\left(1-\frac{1}{4 Q^{2}}\right)^{1 / 2}-\frac{\omega_{0}}{2 Q}\right] T\right\} \\
& +B \exp \left\{\left[-i \omega_{0}\left(1-\frac{1}{4 Q^{2}}\right)^{1 / 2}-\frac{\omega_{0}}{2 Q}\right] T\right\}, \\
a_{y 0}= & C \exp \left\{\left[i \omega_{0}\left(1-\frac{1}{4 Q^{2}}\right)^{1 / 2}-\frac{\omega_{0}}{2 Q}\right] T\right\} \\
& +D \exp \left\{\left[-i \omega_{0}\left(1-\frac{1}{4 Q^{2}}\right)^{1 / 2}-\frac{\omega_{0}}{2 Q}\right] T\right\} .
\end{aligned}
$$

Continuity at $T=0$ implies

$$
a_{x 0}(0+)=a_{x 0}(0-)=e^{-i \omega \tau} a_{x 0}(\tau-),
$$




$$
a_{10}(0+)=a_{y 0}(0-)=e^{-i \omega \tau} a_{y 0}(\tau-),
$$

while Eqs. (57) imply the jump conditions:

$$
\begin{aligned}
& \frac{d a_{x 0}}{d T}(0+)-\frac{d a_{x 0}}{d T}(0-) \\
& =\frac{2 \omega_{0}^{4} \in \tau\left[a_{x 0}(0)+i \omega_{c} a_{y 0}(0) / \Omega\right]}{\omega_{c}^{2}-\Omega^{2}}, \\
& \frac{d a_{y 0}}{d T}(0+)-\frac{d a_{y 0}}{d T}(0-) \\
& =\frac{2 \omega_{0}^{4} \in \tau\left[a_{\not 0}(0)-i \omega_{c} a_{x 0}(0) / \Omega\right]}{\omega_{c}^{2}-\Omega^{2}},
\end{aligned}
$$

where

$$
\frac{d a_{x 0}}{d T}(0-)=e^{-i \omega \tau} \frac{d a_{x 0}}{d T}(\tau-)
$$

and

$$
\frac{d a_{y 0}}{d T}(0-)=e^{-i \omega \tau} \frac{d a_{\not 0}}{d T}(\tau-)
$$

Inserting the ansatz (58) into Eqs. (59) and (60) yields four equations in five unknowns: $A, B, C, D$, and $\Omega$. Eliminating $A, B, C$, and $D$ gives the dispersion relation

$$
\Omega^{2} \pm \omega_{c} \Omega=\frac{\omega_{0}^{3} \epsilon \tau \sin \left[\omega_{0} \sqrt{1-\left(1 / 4 Q^{2}\right)} \tau\right]}{2 \sqrt{1-\left(1 / 4 Q^{2}\right)} \sin \left\{\left[\omega_{0} \sqrt{1-\left(1 / 4 Q^{2}\right)}-\omega+\left(i \omega_{0} / 2 Q\right)\right](\tau / 2)\right\} \sin \left\{\left[-\omega_{0} \sqrt{1-\left(1 / 4 Q^{2}\right)}-\omega+\left(i \omega_{0} / 2 Q\right)\right](\tau / 2)\right\}}
$$

Equation (61) can also be obtained directly from Eq. (54) by letting $\omega_{c} \rightarrow \omega_{c} / 2$ and $\Omega \rightarrow \Omega \pm \omega_{c} / 2$. If the period between bunches $\tau$ is sufficiently small that $\omega_{0} \tau<1$, and we consider frequencies $\omega$ sufficiently small that $\omega \tau<1$, then the right-hand side of Eq. (61) reduces to $-\Gamma$, giving the two-dimensional continuum dispersion relation of $\mathrm{Eq}$. (15). The right-hand side of Eq. (61) vanishes for

$$
\tau=n \pi /\left(\omega_{0} \sqrt{1-1 / 4 Q^{2}}\right)=(n / 2) \tau_{0} .
$$

Thus, BBU growth vanishes when the bunch separation is an integral number of half-periods of the $\mathrm{TM}_{110}$ mode, as in the case of quadrupole focusing.

For frequencies near $\omega_{0}$, obeying $\left|\omega-\omega_{0}\right|$ $\lesssim \omega_{0} / 2 Q$, and bunch separations sufficiently different from $(n / 2) \tau_{0}$, obeying $\left|\tau-(n / 2) \tau_{0}\right|>\tau / 2 Q$, and $n<Q$, the right-hand side of Eq. (61) reduces to $-\Gamma$, so that the two-dimensional continuum dispersion relation applies. Thus, for $\left|\tau-(n / 2) \tau_{0}\right|>\tau / 2 Q$ and $n<Q$, BBU growth is given by the $2 \mathrm{D}$ continuum theory.

In Fig. 3, we plot the growth rate versus the normalized bunch separation for the one- and two-dimensional models [Eqs. (54) and (61)]. Figure 3(a) describes the weak focusing regime, Fig. 3(b) describes a case intermediate between the weak and strong focusing regimes, while Fig. 3(c) describes the strong focusing regime. As in the continuum case, nearly identical growth rates are obtained by the two models in the weak focusing regime, while the two-dimensional model gives twice the growth of the onedimensional model in the strong focusing regime. BBU growth vanishes for $\tau=(n / 2) \tau_{0}$, where $n$ is an integer and $\tau_{0}=2 \pi /\left(\omega_{0} \sqrt{1-1 / 4 Q^{2}}\right)$ is the period of the $T M_{110}$ cavity mode with finite $Q$. In Figs. 3 (a) and $3(\mathrm{~b})$, growth is maximized for $\tau=(n / 2) \tau_{0}(1+1 / 2 Q)$, as in the case of a steady-state $(\omega=0)$ disturbance., ${ }^{6,16}$ This maximum in growth is pronounced when $Q$ is sufficiently large. For $\left|\tau-(n / 2) \tau_{0}\right|>\tau / 2 Q$, BBU growth is the same as that calculated by the continuum models in Fig. 1, as expected.

Finally, we consider solenoidal focusing with perturbed cavities in which the vertical and horizontal polarizations of the $\mathrm{TM}_{110}$ mode have sufficiently different resonant frequencies so that, for $\omega \cong \omega_{x}$, the $y$ polarization is

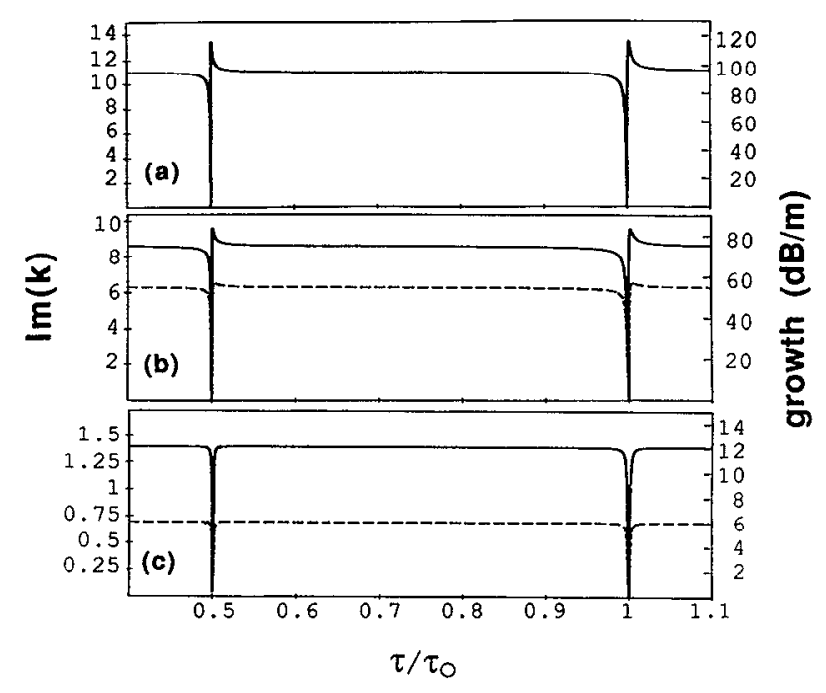

FIG. 3. Graphs of the spatial growth rate $\operatorname{Im}(k)$ as a function of the normalized bunch separation time $\tau / \tau_{0}$. Curves are shown for the onedimensional model (dashed line) and the two-dimensional model (solid line). Growth is shown at an angular frequency $\omega$ for which growth is near maximum. The parameters used are: kinetic energy $=700 \mathrm{keV}$, current $=100 \mathrm{~A}, \mathrm{TM}_{110}$ mode frequency $=2.5 \mathrm{GHz}, Q=200$, and $l / L$ $=0.15$. (a) Growth rates in the weak focusing regime, with a betatron wavelength of $5 \mathrm{~m}$, and $\left|\Gamma\left(\omega_{0}\right)\right| / \omega_{c}^{2}=100 ; \omega=\omega_{0}[1-(1 / 2 \sqrt{3} Q)]$. (b) Growth rates for a case intermediate between the weak and strong focusing regimes, with a betatron wavelength of $0.5 \mathrm{~m}$, and $\left|\Gamma\left(\omega_{0}\right)\right| / \omega_{\mathrm{c}}^{2}=1 ; \omega=\omega_{0}$. (c) Growth rates for a strong focusing case, with a betatron wavelength of $5 \mathrm{~cm}$, and $\left|\Gamma\left(\omega_{0}\right)\right| / \omega_{c}^{2}=0.01 ; \omega=\omega_{0}$. 
not excited. Then, the term $a_{y 0}$ vanishes in Eq. (60a), for $\omega \cong \omega_{x}$. Consequently, Eqs. (59a) and (60a) are the same as Eqs. (52) and (53) of the one-dimensional model. The one-dimensional dispersion relation of Eq. (54) will then apply for $\omega \cong \omega_{x}$.

\section{Pulsed beam with finite pulse length}

Recently, there has been interest in achieving low BBU growth by using a pulsed beam with bunch separation equal to an integral number of half-periods of the $\mathrm{TM}_{110}$ mode. ${ }^{18}$ In this subsection, we consider the finite BBU growth that is expected from finite pulse lengths and bunch separations differing slightly from $(n / 2) \tau_{0}$. We restrict the discussion to quadrupole focusing systems. By letting $\omega_{c} \rightarrow \omega_{\mathcal{C}} / 2$ and $\Omega \rightarrow \Omega \pm \omega_{\mathcal{C}} / 2$, the dispersion relations we obtain will also describe solenoidal focusing with cylindrical symmetry.

For the case of $\delta$-function bunches whose bunch separation is $\tau=(n / 2) \tau_{0}+\delta$, where the timing error $|\delta|<\pi /\left(\omega_{0} \sqrt{1-1 / 4 Q^{2}}\right)=\tau_{0} / 2$, the dispersion relation of Eq. (54) reduces to

$\Omega^{2}-\omega_{c}^{2}= \begin{cases}\frac{\omega_{0}^{4} \epsilon \tau \delta}{2 \sin ^{2}\left\{\left[\omega-\left(i \omega_{0} / 2 Q\right)\right](\tau / 2)\right\}}, & \text { for } n \text { even, } \\ \frac{\omega_{0}^{4} \epsilon \tau \delta}{2 \cos ^{2}\left\{\left[\omega-\left(i \omega_{0} / 2 Q\right)\right](\tau / 2)\right\}}, & \text { for } n \text { odd. }\end{cases}$

This approximation is valid when $\left|\omega_{0} \delta\right|<n \pi / 2 Q$ and $\left|\omega_{0} \delta\right|<1$.

We now calculate an analogous result for the BBU growth resulting from a finite pulse duration $\Delta$, where the quantity $\Delta$ is in units of time. To model a finite pulse length, we consider the model of Eqs. (48) and (49), where the $\delta$ function on the right-hand side of Eq. (49) is replaced by a flattop function: $\delta_{\Delta}(T)=1 / \Delta$ for $-\Delta<T$ $<0$, and zero elsewhere:

$v^{2} \gamma \frac{\partial^{2} x}{\partial Z^{2}}+\gamma \omega_{c}^{2} x=a$,

$$
\left(\frac{\partial^{2}}{\partial T^{2}}+\frac{\omega_{0}}{Q} \frac{\partial}{\partial T}+\omega_{0}^{2}\right) a=2 \gamma \omega_{0}^{4} \epsilon x \tau \sum_{j=-\infty}^{\infty} \delta_{\Delta}(T-j \tau) .
$$

This system is invariant under arbitrary translations in $Z$, and periodic in $T$ with period $\tau$. We thus consider solutions of the form $a=a_{0}(T) e^{i n Z / v}$ where $a_{0}(T)=u(T) e^{i \omega T}$, and $u(T)$ is periodic with period $\tau$. Substituting this solution form into Eqs. (63) and (64) yields

$$
\left(\frac{d^{2}}{d T^{2}}+\frac{\omega_{0}}{Q} \frac{d}{d T}+\omega_{0}^{2}\right) a_{0}=\frac{2 \omega_{0}^{4} \epsilon \tau a_{0}}{\omega_{c}^{2}-\Omega^{2}} \sum_{j=-\infty}^{\infty} \delta_{\Delta}(T-j \tau) .
$$

This equation can be solved in the region $0<T<\tau-\Delta$ to give

$$
a_{0}=A \exp \left\{\left[i \omega_{0}\left(1-\frac{1}{4 Q^{2}}\right)^{1 / 2}-\frac{\omega_{0}}{2 Q}\right] T\right\}
$$

$$
+B \exp \left\{\left[-i \omega_{0}\left(1-\frac{1}{4 Q^{2}}\right)^{1 / 2}-\frac{\omega_{0}}{2 Q}\right] T\right\} .
$$

In the region $-\Delta<T<0$, Eq. (65) is solved by

$$
\begin{aligned}
a_{0}= & C \exp \left[i \omega_{0}\left(1-\frac{1}{4 Q^{2}}-\frac{2 \omega_{0}^{2} \epsilon \tau}{\Delta\left(\omega_{c}^{2}-\Omega^{2}\right)}\right)^{1 / 2}-\frac{\omega_{0}}{2 Q}\right] T \\
& +D \exp \left[-i \omega_{0}\left(1-\frac{1}{4 Q^{2}}-\frac{2 \omega_{0}^{2} \epsilon \tau}{\Delta\left(\omega_{c}^{2}-\Omega^{2}\right)}\right)^{1 / 2}\right. \\
& \left.-\frac{\omega_{0}}{2 Q}\right] T .
\end{aligned}
$$

At the times $T=0$ and $T=-\Delta, a_{0}$ and $d a_{0} / d T$ are continuous. Using the relations $a_{0}(-\Delta-)$ ) $=e^{-i \omega \tau} a_{0}(\tau-\Delta-)$ and

$$
\frac{d a_{0}}{d T}(-\Delta-)=e^{-i \omega \tau} \frac{d a_{0}}{d T}(\tau-\Delta-),
$$

we obtain four equations in the unknowns $A, B, C, D$, and $\Omega$. Eliminating $A, B, C$, and $D$, we obtain the equation

$$
\begin{aligned}
0= & (M-P)(R-S)\left(e^{(P+M) \tau-(P+M) \Delta}\right. \\
& \left.+e^{2 i \omega \tau-(P+M) \Delta}\right)-(R-P)^{2}\left(e^{(P+i \omega) \tau-(P+R) \Delta}\right. \\
& \left.+e^{(M+i \omega) \tau-(M+S) \Delta}\right)+(S-P)^{2} \\
& \times\left(e^{(P+i \omega) \tau-(P+S) \Delta}+e^{(M+i \omega) \tau-(M+R) \Delta}\right),
\end{aligned}
$$

where

$$
\begin{aligned}
P= & i \omega_{0}\left(1-1 / 4 Q^{2}\right)^{1 / 2}-\left(\omega_{0} / 2 Q\right) \\
M= & -i \omega_{0}\left(1-1 / 4 Q^{2}\right)^{1 / 2}-\left(\omega_{0} / 2 Q\right) \\
R= & i \omega_{0}\left\{1-1 / 4 Q^{2}-2 \omega_{0}^{2} \epsilon \tau /\left[\Delta\left(\omega_{c}^{2}-\Omega^{2}\right)\right]\right\}^{1 / 2} \\
& -\left(\omega_{0} / 2 Q\right),
\end{aligned}
$$

and

$$
\begin{aligned}
S= & -i \omega_{0}\left\{1-1 / 4 Q^{2}-2 \omega_{0}^{2} \epsilon \tau /\left[\Delta\left(\omega_{c}^{2}-\Omega^{2}\right)\right]\right\}^{1 / 2} \\
& -\left(\omega_{0} / 2 Q\right) .
\end{aligned}
$$

Defining the frequencies $\omega_{1}=\omega_{0}\left(1-1 / 4 Q^{2}\right)^{1 / 2}$ and

$$
\omega_{2}=\omega_{0}\left\{1-1 / 4 Q^{2}-2 \omega_{0}^{2} \epsilon \tau /\left[\Delta\left(\omega_{c}^{2}-\Omega^{2}\right)\right]\right\}^{1 / 2},
$$

Eq. (68) gives the dispersion relation

$$
\begin{aligned}
0= & 4 \omega_{1} \omega_{2} \cos \left[\omega-\left(i \omega_{0} / 2 Q\right)\right] \tau \\
& +\left(\omega_{1}-\omega_{2}\right)^{2} \cos \left[\omega_{1} \tau-\left(\omega_{1}+\omega_{2}\right) \Delta\right] \\
& -\left(\omega_{1}+\omega_{2}\right)^{2} \cos \left[\omega_{1} \tau-\left(\omega_{1}-\omega_{2}\right) \Delta\right] .
\end{aligned}
$$

For a short pulse duration, $\Delta<\tau_{0} / 2$, and bunch separation close to an integral number of half-cavity periods,

$$
\tau=n \pi /\left(\omega_{0} \sqrt{1-1 / 4 Q^{2}}\right)+\delta=(n / 2) \tau_{0}+\delta,
$$

where $|\delta|<\tau_{0}$, the last two cosines in Eq. (69) can be expanded through fourth order to yield an approximate dispersion relation for the BBU instability: 
$\Omega^{2}-\omega_{c}^{2}=\frac{\omega_{0}^{4} \epsilon \tau}{\xi}\left[-\delta \pm\left(\delta^{2}+\frac{2 \xi \delta \Delta}{3}-\frac{\xi \Delta^{2}}{3}\right)^{1 / 2}\right]$,

where

$$
\xi=-2\left(1-(-1)^{n} \cos \left\{\left[\omega-\left(i \omega_{0} / 2 Q\right)\right] \tau\right\}\right) .
$$

Equation (70) is approximately obeyed for sufficiently small timing errors and pulse lengths satisfying $\left|\omega_{0} \delta\right|$ $<n \pi / 2 Q<1 / 2 \sqrt{3}$, and $\left|\omega_{0} \Delta\right|<1$. Because $n \pi \cong \omega_{0} \tau$, the $\mathrm{TM}_{110}$ mode must not decay significantly between pulses. In addition, $|\xi|<1$ is required, which is satisfied when $\left|\omega-\omega_{0}\right|<\omega_{0} / 2 n \pi \sqrt{3}$. This is not a severe restriction since frequencies near $\omega_{0}$ give the greatest BBU growth and thus are of primary interest. For $\Delta=0$ (vanishing pulselength), Eq. (70) gives the BBU dispersion relation of Eq. (62), as expected. In addition, the nongrowing roots $\Omega^{2}-\omega_{c}^{2}=0$ occur. For $\delta=0$ (no timing error), Eq. (70) becomes

$$
\begin{aligned}
\Omega^{2}-\omega_{c}^{2} & \begin{cases} \pm \frac{\omega_{0}^{4} \epsilon \tau \Delta}{2 \sqrt{3} \sin \left\{\left[\omega-\left(i \omega_{0} / 2 Q\right)\right](\tau / 2)\right\}}, & \text { for } n \text { even, } \\
\pm \frac{\omega_{0}^{4} \epsilon \tau \Delta}{2 \sqrt{3} \cos \left\{\left[\omega-\left(i \omega_{0} / 2 Q\right)\right](\tau / 2)\right\}}, & \text { for } n \text { odd }\end{cases}
\end{aligned}
$$

Equation (71) shows that BBU growth occurs in the absence of a timing error because of the finite pulse duration of the electron bunches. Electron bunches of finite pulsewidth $\Delta$ can also be modeled by "head" and "tail" macroparticles rather than a flattop pulse. ${ }^{18}$ In this approach, the flattop $\left(\delta_{\Delta}\right)$ term in Eq. (64) is replaced by $[\delta(T-j \tau)$ $+\delta(T-j \tau+\Delta)] / 2$. For zero timing error, we obtain a dispersion relation identical to Eq. (71) with $\Delta \rightarrow \sqrt{3} \Delta$. Increased BBU growth results from the concentration of current at the head and tail of the pulse.

In the strong focusing regime, maximum growth occurs for $\omega \cong \omega_{0}$. At this frequency, Eq. (71) becornes

$$
\Omega^{2}-\omega_{c}^{2}= \pm(2 \pi / \sqrt{3})\left(\Delta / \tau_{0}\right) \Gamma\left(\omega_{0}\right) .
$$

In comparison with the continuum model of Eq. (3), $\Gamma$ is multiplied by the factor $(2 \pi / \sqrt{3})\left(\Delta / \tau_{0}\right)$, so that BBU growth is modified by this factor for pulses separated by $(n / 2) \tau_{0}$ in the strong focusing regime. Thus, for $\left(\Delta / \tau_{0}\right)<1$, significant BBU growth reduction can be obtained by the use of a pulsed current.

In Fig. 4 we display the results of Eq. (70) for the parameters of Fig. 3 and two different nonzero pulse lengths. We also show the results of Eq. (54) for $\delta$-function pulses. The finite pulse lengths result in nonzero BBU growth for all values of pulse spacing $\tau$, and a shift in the value of $\tau$ that gives minimum BBU growth. With a finite pulse length, $\tau=(n / 2) \tau_{0}$ no longer gives minimum BBU growth, so that a slight timing adjustment may be advantageous in applications using a pulsed beam.

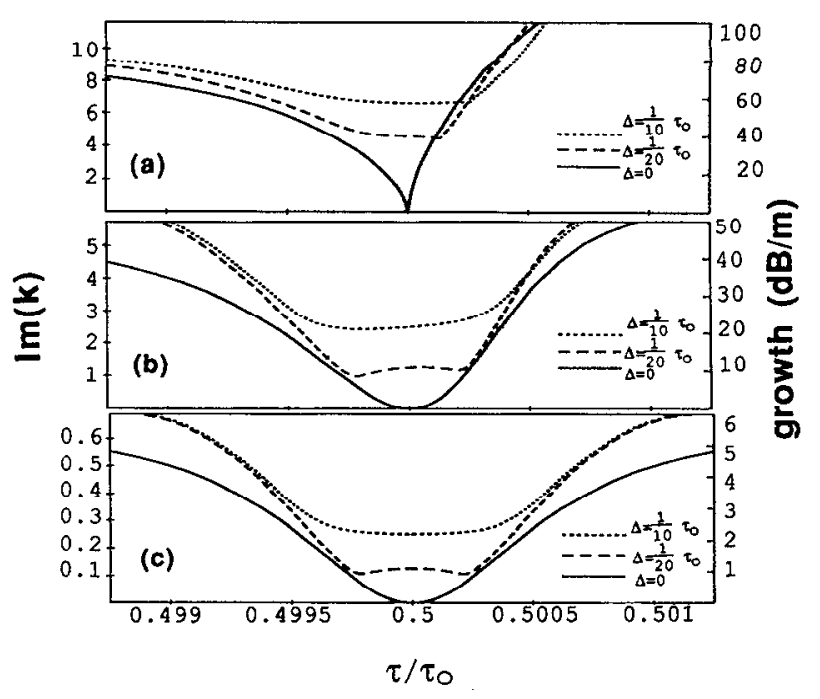

FIG. 4. Graphs of the spatial growth rate $\operatorname{Im}(k)$ as a function of normalized bunch separation time $\tau / \tau_{0}$ for different values of pulse length $\Delta$. Growth is shown at an angular frequency $\omega$ for which growth is near maximum. Curves are shown for pulselengths of $\Delta=0$ (solid black line), $\Delta=\frac{1}{20} \tau_{0}$ (dashed line), and $\Delta=\frac{1}{10} \tau_{0}$ (dotted line). The parameters used are: kinetic energy $=700 \mathrm{keV}$, current $=100 \mathrm{~A}, \mathrm{TM}_{110}$ mode frequency $=2.5 \mathrm{GHz}, Q=200$, and $l / L=0.15$. (a) Growth rates in the weak focusing regime, with a betatron wavelength of $5 \mathrm{~m}$, and $\left|\Gamma\left(\omega_{0}\right)\right| / \omega_{c}^{2}$ $=100 ; \omega=\omega_{0}[1-(1 / 2 \sqrt{3} Q)]$. (b) Growth rates for a case intermediate between the weak and strong focusing regimes, with a betatron wavelength of $0.5 \mathrm{~m}$, and $\left|\Gamma\left(\omega_{0}\right)\right| / \omega_{c}^{2}=1 ; \omega=\omega_{0}$. (c) Growth rates in the strong focusing regime, with a betatron wavelength of $5 \mathrm{~cm}$, and $\left|\Gamma\left(\omega_{0}\right)\right| / \omega_{c}^{2}=0.01 ; \omega=\omega_{0}$.

\section{SUMMARY}

Dispersion relations for the BBU instability have been derived for quadrupole and solenoidal focusing. We first considered a continuum model appropriate for constant beam current and cavity spacings small compared to the betatron wavelength and $e$ folding length of BBU growth. The $x-y$ coupling of solenoidal focusing affects the BBU growth rate for cylindrical pillbox accelerator cavities, in which the $\mathrm{TM}_{110}$ frequency is independent of polarization. BBU growth in the strong focusing regime is twice that of a quadrupole focusing system with the same betatron wavelength. By perturbing the cavities so that the vertical and horizontal polarizations of the $\mathrm{TM}_{110}$ mode have sufficiently different resonant frequencies, this growth may be halved.

A model was presented for the case where the spacing of the accelerator cavities can be large compared to the betatron wavelength and the $e$ folding length of BBU growth. We first neglected the finite length of the cavities. For quadrupole focusing, BBU growth vanishes when the spacing is an integral multiple of one-half betatron wavelength. With solenoidal focusing and cylindrical pillbox cavities, the cavity spacing must be an integral multiple of the betatron wavelength to prevent BBU growth. Finally, we considered the effect of finite cavity length with quadrupole focusing, showing that the BBU growth does not completely vanish for spacings that are integral multiples of one-half betatron wavelength. For such spacings in the 
strong focusing regime, BBU growth is modified by the factor $(2 \pi / \sqrt{3})\left(l / \lambda_{b}\right)$ when compared to the continuum model, where $l$ is the cavity length, $\lambda_{b}$ is the betatron wavelength, and $\left(l / \lambda_{b}\right)<1 / 2 \pi$.

A model was also presented for a periodically pulsed electron-beam current. We first considered vanishing pulselength by modeling $\delta$-function current pulses. BBU growth vanishes when the pulse separation is a multiple of one-half of the $\mathrm{TM}_{110}$ period, for both quadrupole and solenoidal focusing. When a finite pulselength was considered, the BBU growth did not completely vanish. For pulse separations that are a multiple of one-half of the $\mathrm{TM}_{110}$ period in the strong focusing regime, BBU growth is modified by the factor $(2 \pi / \sqrt{3})\left(\Delta / \tau_{0}\right)$ when compared to the continuum model, where $\Delta$ is the pulse duration, $\tau_{0}$ is the period of the $\mathrm{TM}_{110}$ mode, and $\left(\Delta / \tau_{0}\right)<1 / 2 \pi$.

Because the $e$ folding length, wavelength, and group velocity of the $\mathrm{BBU}$ instability are directly calculable from the dispersion relation, the formulas presented here may be used to calculate these important quantities under a broad range of conditions.

\section{ACKNOWLEDGMENTS}

We appreciate valuable discussions with Y. Y. Lau. This research was supported by the Strategic Defense Initiative through an Office of Naval Research contract.

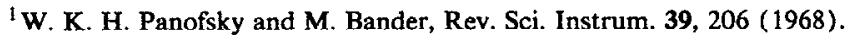

${ }^{2}$ R. Helm and G. Loew, "Beam Breakup," in Linear Accelerators, edited by P. M. Lapostelle and A. L. Septier (North-Holland, Amsterdam, 1970), Chap. B.1.4, p. 173.

${ }^{3}$ V. K. Neil and R. K. Cooper, Part. Accel. 1, 111 (1970).

${ }^{4}$ V. K. Neil, L. S. Hall, and R. K. Cooper, Part. Accel. 9, 213 (1979).

${ }^{5}$ A. W. Chao, B. Richter, and C. Y. Yao, Nucl. Instrum. Methods 178, 1 (1980).

${ }^{6}$ R. L. Gluckstern, R. K. Cooper, and P. J. Channell, Part. Accel. 16, 125 (1985).

${ }^{7}$ Y. Y. Lau, Phys. Rev. Lett. 63, 1141 (1989).

${ }^{8}$ S. Humphries, Jr., Principles of Charged Particle Acceleration (Wiley, New York, 1986).

${ }^{9}$ R. A. Bosch and R. M. Gilgenbach, Appl. Phys. Lett. 58, 699 (1991).

${ }^{10}$ P. R. Menge, R. A. Bosch, R. M. Gilgenbach, J. J. Choi, H. Ching, and T. A. Spencer, Proc. SPIE 1407, 578 (1991).

${ }^{11}$ R. M. Gilgenbach, P. R. Menge, R. A. Bosch, J. J. Choi, H. Ching, and T. A. Spencer, in Proceedings of the 1991 IEEE Particle Accelerator Conference (IEEE, New York, 1991).

${ }^{12}$ Y. Y. Lau, NRL Memo Report No. 6237, 1988.

${ }^{13}$ P. Allison, M. J. Burns, G. J. Caporaso, and A. G. Cole, in Proceedings of the 1991 IEEE Particle Accelerator Conference (IEEE, New York, 1991); also available as Los Alamos Report LA-UR-90-4224.

${ }^{14}$ Y. Y. Lau (private communication).

${ }^{15}$ Y. Y. Lau and D. G. Colombant, Appl. Phys. Lett. 55, 2673 (1989).

${ }^{16}$ C. L. Bohn and J. R. Delayen, Proc. SPIE 1407, 566 (1991).

${ }^{17}$ J. R. Delayen and C. L. Bohn, Proceedings of the 1991 IEEE Particle Accelerator Conference (IEEE, New York, 1991).

${ }^{18}$ K. A. Thompson and R. D. Ruth, Phys. Rev. D 41, 964 (1990). 